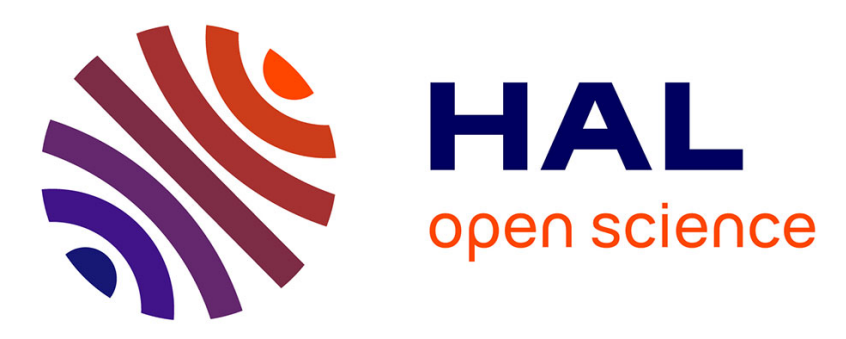

\title{
Hepatic Stellate Cell Senescence in Liver Tumorigenesis
}

Nadine Martin, Dorian Ziegler, Romain Parent, David Bernard

\section{To cite this version:}

Nadine Martin, Dorian Ziegler, Romain Parent, David Bernard. Hepatic Stellate Cell Senescence in Liver Tumorigenesis. Hepatology, In press, 10.1002/hep.31556 . hal-03008992

\section{HAL Id: hal-03008992 \\ https://hal.science/hal-03008992}

Submitted on 17 Nov 2020

HAL is a multi-disciplinary open access archive for the deposit and dissemination of scientific research documents, whether they are published or not. The documents may come from teaching and research institutions in France or abroad, or from public or private research centers.
L'archive ouverte pluridisciplinaire HAL, est destinée au dépôt et à la diffusion de documents scientifiques de niveau recherche, publiés ou non, émanant des établissements d'enseignement et de recherche français ou étrangers, des laboratoires publics ou privés. 
HEPATOLOGY Elsewhere

\section{Hepatic Stellate Cell Senescence in Liver Tumorigenesis}

Nadine Martin ${ }^{1, *}$, Dorian V Ziegler ${ }^{1}$, Romain Parent ${ }^{1}$, David Bernard ${ }^{1, *}$

${ }^{1}$ Centre de Recherche en Cancérologie de Lyon, Inserm U1052, CNRS UMR5286, Université de Lyon, Centre Léon Bérard, Lyon, France.

*Correspondence: nadine.martin@lyon.unicancer.fr and david.bernard@lyon.unicancer.fr 


\section{MAIN TEXT}

Hepatocellular carcinoma (HCC) is the most common primary liver cancer and one of the main causes of cancer-related deaths. HCC is mainly promoted by chronic viral hepatitis B and C, alcohol, non-alcoholic fatty liver disease (NAFLD) induced by obesity, as well as several genetic diseases of the liver. Due to the intricacy and heterogeneity of risk factors, microenvironment and genetic alterations associated with HCC, the dissection of molecular players and cellular processes underlying tumor development is still challenging (1). In a recent paper (Li F, Huangyang P, Burrows M et al. FBP1 loss disrupts liver metabolism and promotes tumorigenesis through a hepatic stellate cell senescence secretome. Nat Cell Biol. 2020 Nat Cell Biol 2020;22(6):728-739), Li et al identified the gluconeogenic enzyme fructose 1,6-bisphosphatase 1 (FBP1) as a tumor suppressor in the liver and present evidences using senolytic compounds (compounds inducing death of senescent cells) that senescence of hepatic stellate cells (HSCs) induced by loss of FBP1 promotes HCC in mice (2).

Li et al first observed that FBP1 expression is silenced during HCC progression, both in human tumors from the TCGA database and murine tumors from Trp53 floxflox mice, diethylnitrosamine (DEN)-treated mice and NAFLD-HCC murine models. The authors then took advantage of a conditional Fbp $1^{\text {flox/flox }}$ allele to study the effects of specific FBP1 deletion in hepatocytes. FBP1 loss disturbed liver metabolism, as evidenced by abolished gluconeogenesis, defective fatty acid oxidation, hepatomegaly and mild hepatic steatosis. Moreover, this loss triggered fibrosis and accelerated tumor initiation and progression in three distinct murine models of liver tumors: (i) HCC induced by DEN treatment or by a combination of western diet and $\mathrm{CCl}_{4}$ treatment and (ii) intrahepatic cholangiocarcinoma induced by hepatic p53 deletion. As fibrosis is known to result from activation of HSCs, the authors then focused on investigating their role in the observed pro-tumoral effect of FBP1 deletion. Hepatocytic 
loss of FBP1 induced HSC activation coupled to an initial pro-fibrotic effect, followed by their senescence and their switch to a pro-inflammatory senescence-associated secretory phenotype (SASP). Dasatinib and Quercetin combination or Navitoclax senolytics were then used to explore the potential benefit of killing senescent HSCs. After validating in vitro the toxicity of these compounds towards senescent HSCs, Li et al observed that these treatments dampened tumor progression induced by hepatocytic FBP1 deletion. Finally, they proposed high mobility group protein 1 (HMGB1) as a mediator of the crosstalk between FBP1-deficient hepatocytes and HSCs.

Senescence is a cellular response to stresses (telomere shortening, oxidative stress...) which can be induced in an autocrine or paracrine manner. In addition of being cell cycle arrested, senescent cells secrete numerous factors, including pro-inflammatory factors, which impact the microenvironment (3). In the context of liver cancer, HSC senescence was previously shown to promote obesity-associated HCC development through the SASP, using mice harboring a knockout for the SASP component and inducer IL-1 $\beta$ or depleted in HSCs (4). Li et al further highlighted the functional role for HSC senescence in HCC development using other experimental models (in the context of loss of FBP1) and demonstrated it for the first time using senolytic compounds. Of note, Wakita et al also showed that a newly identified senolytic compound (BET family protein degrader) reduces obesity-associated HCC development in mice (5). Altogether, these articles support a key role of HSC senescence in promoting liver tumorigenesis. Still, as senolytics used in these studies are not specific of senescent HSCs and depletion of p53 in HSCs was reported to decrease HSC senescence and increase liver tumorigenesis (6), the role of HSC senescence in hepatocarcinogenesis remains controversial. 
Li et al documented senescence of activated HSCs with several senescence markers, some of which were shown to colocalize with markers of HSCs (CD140B) or activated HSCs ( $\alpha$-SMA). Initially fibrogenic, activated HSCs were previously reported to acquire proinflammatory properties by entering senescence during liver fibrosis. However, the identification of components and regulators of the SASP in the last years unraveled that this secretome is multifaceted and triggers other effects aside from the pro-inflammatory ones, including cell plasticity, regeneration and stemness (3). In this context, a more detailed characterization of the SASP of senescent HSCs and of its functional impact could improve our understanding of their role in liver tumorigenesis.

In an effort to understand the molecular mechanisms underlying the effects of hepatic FBP1 depletion, the authors performed secretome profiling of FBP1-deleted hepatocytes and focused on the observed increased release of HMGB1. They used inflachromene (ICM), a molecule shown to block this secretion, to assess the functional role of HMGB1 and observed an inhibition of HSC activation, subsequent HSC senescence and tumorigenesis. ICM can impact all cell types and is known to also impair autophagy and in another context, HMGB1 deletion in liver had no impact on inflammation and fibrosis (7). Hence, further specific assessment of the role of hepatocyte-secreted HMGB1 would be needed, using genetic tools. Moreover, the mechanisms downstream of HMGB1 as well as the involvement of other components of the hepatocytic secretome remain to be explored to fully understand the crosstalk between FBP1-deficient hepatocytes and HSCs. Interestingly, several proteins identified in the secretome of FBP1-deleted hepatocytes, including HMGB1, CCN1, CCL2, CCL7, IGFBP1 and GDF15, have previously been described as SASP components (3). Moreover, FBP1-deleted hepatocytes display marks of unfolded protein response and endoplasmic reticulum (ER) stress, evidenced by transcriptomic profiling and ER morphology, which may be associated with senescence. Altogether, these findings suggest that FBP1 loss in 
hepatocytes could induce senescence in these cells, resulting in a SASP-dependent communication with HSCs involving HMGB1 and other factors as mentioned above. The potential induction of senescence in hepatocytes and its functional implication in liver tumorigenesis remains an open question.

Hepatic fibrosis is considered as a key contributor to HCC, and activated HSCs as critical mediators by promoting fibrosis and inflammation (1, 8). Interestingly, Li et al by administrating a combination of Dasatinib and Quercetin suppressed progression of liver tumors in DEN-treated mice without improving liver fibrosis, supporting the idea that liver fibrosis is not sufficient to drive HCC and that entry of activated HSCs into senescence is needed (2). A question raised by these findings is whether fibrosis is a required step to promote HCC formation and progression or whether it is a mark of HSC activation, persistent HSC activation being required for their entry into senescence and the promotion of inflammation by the SASP, which could then promote HCC. This pro-inflammatory SASP could also reinforce senescence in its environment and especially in hepatocytes, potentially creating a vicious circle between senescent hepatocytes and senescent HSCs and long-term tumor promotion. Additional experiments using single cell analysis at early and late time points in the tumorigenesis process will be helpful to prove the existence of this feedforward loop.

To conclude, Li et al present novel insights into the interplay between hepatocytes and HSCs (Fig.1), which improve our understanding of the biology of HCC and particularly of the role of senescence in this context. Moreover, although the identification of senolytic drugs displaying high specificity and efficacy remains a major challenge, the authors provide evidence that targeting senescent HSCs could be a promising therapeutic approach for HCC. More generally, this study reinforces the idea that targeting senescent cells in the tumor microenvironment could be in some contexts a valuable therapeutic strategy. Of importance, 
eliminating senescent cells could also be a relevant preventive approach in cirrhotic patients to decrease inflammation and prevent development of HCC. 


\section{ACKNOWLEDGEMENTS}

We thank INCA, INSERM and CNRS for their support.

\section{CONFLICT OF INTEREST}

None declared. 


\section{REFERENCES}

1. Villanueva A. Hepatocellular Carcinoma. N Engl J Med 2019 Apr 11;380(15):14501462.

2. Li F, Huangyang P, Burrows M, Guo K, Riscal R, Godfrey J, et al. FBP1 loss disrupts liver metabolism and promotes tumorigenesis through a hepatic stellate cell senescence secretome. Nat Cell Biol 2020 Jun;22(6):728-739.

3. Gorgoulis V, Adams PD, Alimonti A, Bennett DC, Bischof O, Bishop C, et al. Cellular Senescence: Defining a Path Forward. Cell 2019 Oct 31;179(4):813-827.

4. Yoshimoto S, Loo TM, Atarashi K, Kanda H, Sato S, Oyadomari S, et al. Obesityinduced gut microbial metabolite promotes liver cancer through senescence secretome. Nature 2013 Jul 4;499(7456):97-101.

5. Wakita M, Takahashi A, Sano O, Loo TM, Imai Y, Narukawa M, et al. A BET family protein degrader provokes senolysis by targeting NHEJ and autophagy in senescent cells. Nat Commun 2020 Apr 22;11(1):1935.

6. Lujambio A, Akkari L, Simon J, Grace D, Tschaharganeh DF, Bolden JE, et al. Noncell-autonomous tumor suppression by p53. Cell 2013 Apr 11;153(2):449-460.

7. Khambu B, Huda N, Chen X, Antoine DJ, Li Y, Dai G, et al. HMGB1 promotes ductular reaction and tumorigenesis in autophagy-deficient livers. J Clin Invest 2018 Jun 1;128(6):2419-2435.

8. Coulouarn C, Clement B. Stellate cells and the development of liver cancer: therapeutic potential of targeting the stroma. J Hepatol 2014 Jun;60(6):1306-1309. 


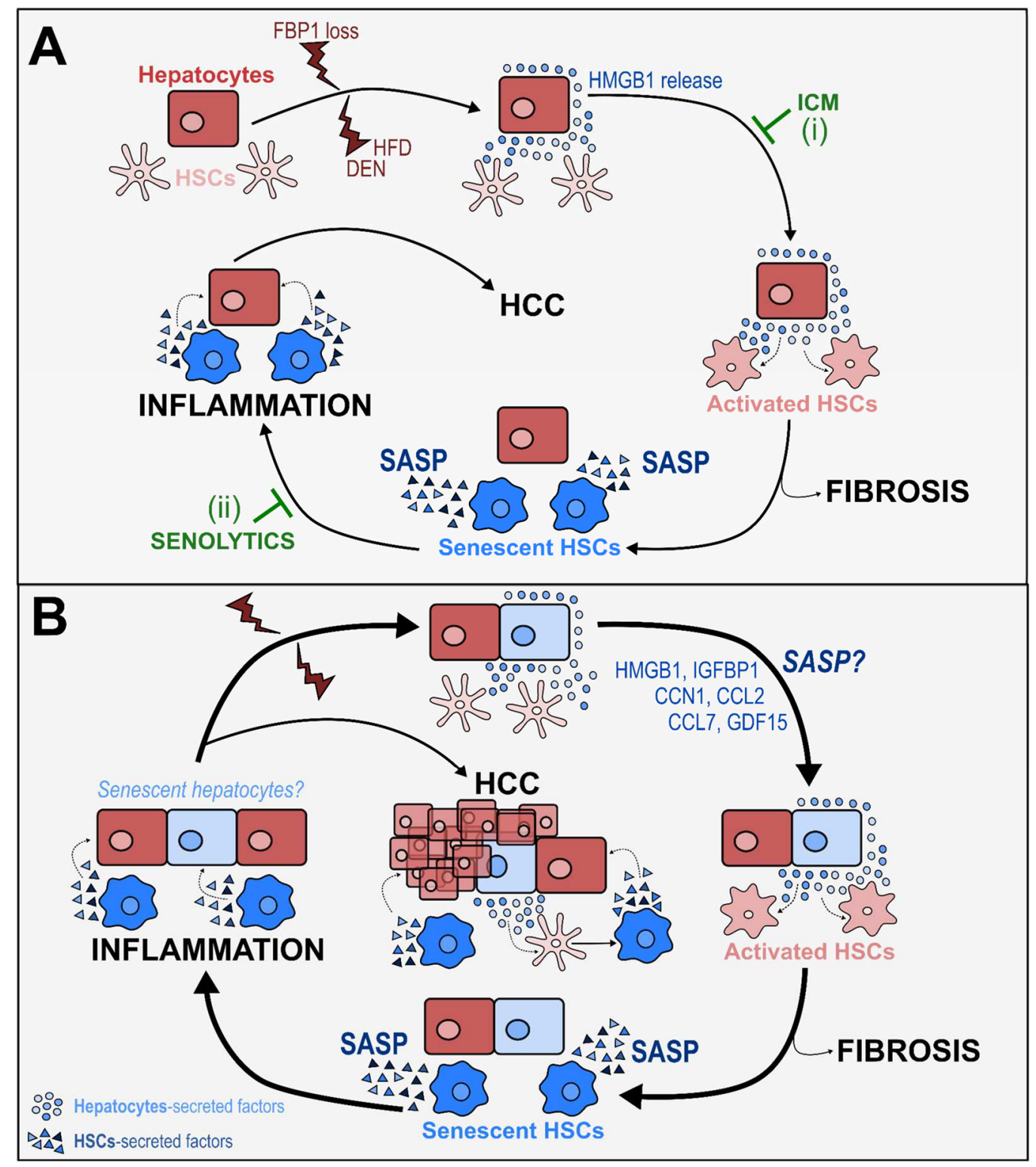

Role of senescence in the crosstalk between hepatocytes and HSCs in liver tumorigenesis

(A) Summary of authors results: FBP1 loss in hepatocytes, HFD or DEN initiate the secretion of factors such as HMGB1. HMGB1 triggers the activation of surrounding HSCs and fibrosis. Unsolved fibrosis and persistent activation of HSCs induce their senescence. Senescent HSCs secrete pro-inflammatory SASP factors, promoting inflammation and HCC. HMGB1 inhibition by Inflachromene (ICM) or senolytic treatment prevents inflammation and HCC. (B) 
Hypothetic model beyond authors' results: The secretome of FBP1-deficient hepatocytes is enriched in HMGB1, IGFBP1, CCN1, CCL2, CCL7 and GDF15 which are SASP factors, raising the possibility that FBP1 loss, HFD and DEN initiate hepatocyte senescence. Their SASP could then trigger HSC activation, exhaustion and finally senescence. Senescent HSCs, by releasing a pro-inflammatory SASP, could in turn reinforce hepatocyte senescence, initiating and amplifying a vicious cycle. The SASP of both hepatocytes and HSCs could reinforce senescence as well as promote cell plasticity, regeneration and stemness especially in cancer cells. 